\title{
ARTROSCOPIA NO TRATAMENTO DA TENDINITE CALCÁRIA REFRATÁRIA DO OMBRO
}

\author{
ARTHROSCOPY IN THE TREATMENT OF REFRACTORY \\ CALCIFIC TENDONITIS OF THE SHOULDER
}

Marcos Rassi Fernandes', Rui José Fernandes ${ }^{2}$

\section{RESUMO}

Objetivos: Analisar os resultados do tratamento artroscópico de pacientes com tendinite calcária do ombro. Métodos: Entre setembro de 2001 e junho de 2006, 55 pacientes com tendinite calcária do ombro, resistentes ao tratamento conservador, foram avaliados, com seguimento de 12 a 70 meses. A média de idade foi de 42 anos, variando de 30 a 64 anos; 44 pacientes eram do sexo feminino (80\%), sendo 37 ombros direitos (67,27\%), com dominância em $63,63 \%$ dos casos. A dor foi o principal sintoma, sendo que o tempo decorrido entre o início dos sintomas e a artroscopia foi em média de 38 meses (cinco a 120 meses). Quanto aos tendões acometidos, o supraespinal em 42 casos, o infraespinal em 11 e a associação entre eles em dois casos. A acromioplastia foi realizada em apenas 12 casos (21,82\%), enquanto a bursectomia subacromial, em todos os casos. Resultados: Segundo os critérios da UCLA, houve 46 casos excelentes e seis bons, totalizando 52 resultados satisfatórios (94,54\%). Conclusão: O tratamento artroscópico da tendinite calcária do ombro sugere ter altos índices de resultados satisfatórios, sendo um método eficaz. A acromioplastia associada não se faz necessária.

Descritores - Ombro/lesões; Ombro/cirurgia; Artroscopia/instrumentação; Tendinopatia; Diagnóstico por imagem

\section{ABSTRACT}

Objective: To evaluate the results of arthroscopic treatment in patients with calcific tendonitis of the shoulder. Methods: Between September 2001 and June 2006, 55 patients with calcific tendonitis of the shoulder that was resistant to prior conservative treatment were evaluated, with follow-up of 12 to 70 months. The mean age was 42 years, ranging from 30 to 64 years; 44 patients were female (80\%), with 37 right shoulders, with a predominance of $63.63 \%$. Pain was the main symptom, and the mean time between onset of symptoms and arthroscopy was 38 months (five to 120 months). In relation to the affected tendons, the supraspinatus tendon was predominant, with 42 cases, the infraspinatus in 11, and an association between the two in 2 cases. Acromioplasty was carried out in 12 patients (21.82\%) and subacromial bursectomy was performed in all cases. Results: According to the UCLA evaluation system, 46 cases were classified as excellent and 6 as good, making a total of 52 satisfactory results achieved (94.54\%). Conclusion: Arthroscopic treatment of calcific tendonitis of the shoulder appears to be an effective method, with high rates of satisfactory results. Associated acromioplasty is not necessary.

Keywords - Shoulder/lesions; Shoulder/surgery; Arthroscopy/ instrumentation; Tendinopathy; Diagnostic imaging

\section{INTRODUÇÃO}

A tendinite calcária é uma das afecções mais comuns do manguito rotador, acometendo, principalmente, pacientes entre 30 e 50 anos, com incidência maior no sexo feminino $^{(1,2)}$. A etiologia ainda permanece desconhecida.

Os depósitos calcários se originam com mais frequência na porção do manguito que suporta as forças de maior intensidade e que mais frequentemente apresenta alterações degenerativas: o tendão do supraespinal ${ }^{(3)}$. Codman chamou essa região de zona crítica do manguito rotador, local onde o suprimento sanguíneo é pobre e corresponde à área de maior pressão e impacto na elevação anterior do braço. Portanto, a localização mais comum da calcificação é na face bursal do tendão supraespinal ${ }^{(4)}$, próximo à sua inserção na grande tuberosidade, motivo pelo

1 - Médico Assistente do Grupo de Ombro e Cotovelo do Hospital Ortopédico de Goiânia e Hospital Geral de Goiânia.

2 - Médico Assistente do Grupo de Ombro e Cotovelo do Hospital Ortopédico de Goiânia.

Trabalho desenvolvido no Grupo de Ombro e Cotovelo do Hospital Ortopédico de Goiânia.

Correspondência: Avenida Azaleias, Qd. 10, Lt. 20, Residencial Jardins Viena - 74935-187 - Aparecida de Goiânia, GO, Brasil. E-mail: marcosombro@ig.com.br 
qual os procedimentos cirúrgicos, quando necessários, acontecem no espaço subacromial. Outras localizações adicionais são os tendões do infraespinal e do subescapular, podendo haver associação entre eles ${ }^{(3,4)}$.

Bosworth $^{(5)}$ realizou uma pesquisa em um grupo não selecionado de 6.061 trabalhadores de uma companhia de seguro de vida com radiografias dos dois ombros e encontrou incidência de 2,7\% de depósitos calcários. Desses, 35\% dos pacientes haviam apresentado sintomas anteriormente. É possível, então, a presença de calcificações em pessoas assintomáticas, em conclusão semelhante à dos estudos de Welfling et $a l^{(6)}$ e Sandstrom e Wahlgreen $^{(7)}$, que encontraram $7,5 \%$ e $20 \%$, respectivamente. Não existem evidências correlacionando os depósitos calcários no ombro com a presença de cálculos em órgãos como rins e vesícula biliar, bem como doenças osteometabólicas ${ }^{(1)}$.

O depósito de cálcio normalmente é intratendíneo ${ }^{(1,3,4)}$ e sofre evolução com definida progressão, segundo Uhthoff e Sarkar ${ }^{(8)}$, passando por fase pré- calcificante, fase formativa e de reabsorção (Figuras 1 e 2), remodelando, posteriormente, para tendão normal. Acredita-se que o tempo dessa remodelação seja a verdadeira variável nesse processo.

O conhecimento da sequência cronológica dessas três fases se torna importante no planejamento terapêutico. Por ser autolimitada, a tendinite calcária deve ser tratada, inicialmente, de modo conservador com antiinflamatórios e reabilitação. A progressão dos sintomas e a ausência de melhora da dor são indicações para outras modalidades de tratamento: aspiração percutânea ${ }^{(3)}$, ondas de choque ${ }^{(9)}$, remoção cirúrgica aberta ${ }^{(1,3,8)}$ ou $\operatorname{artroscópica~}^{(2,10-15)}$.

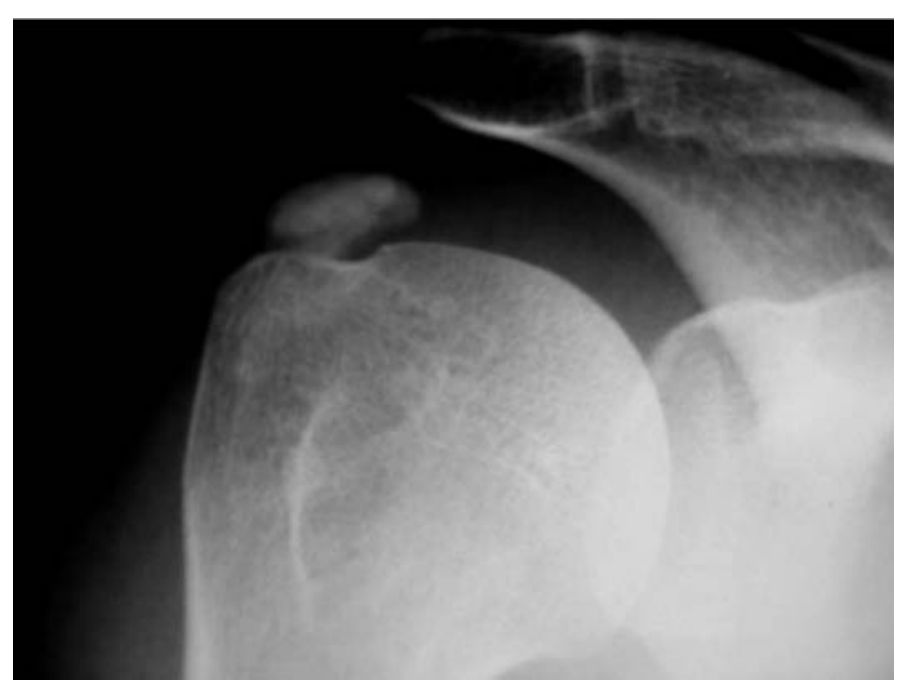

Figura 1 - Fase formativa, segundo Uhthoff

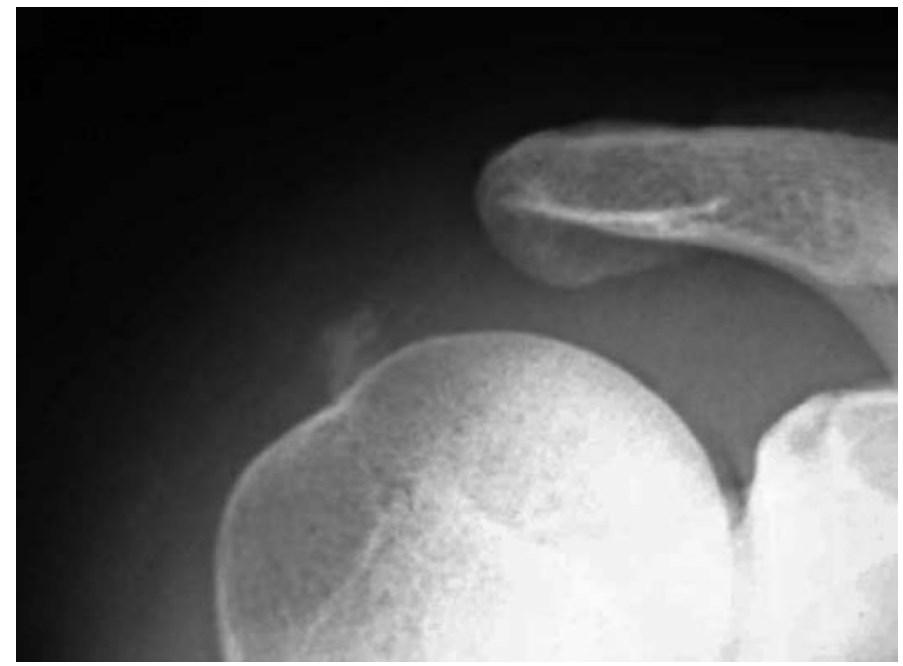

Figura 2 - Tendinite calcária - fase reabsortiva, segundo Uhthoff

O tratamento artroscópico permite completa avaliação articular, reabilitação precoce e evita as complicações descritas com a cirurgia aberta, como os problemas com a inserção do músculo deltoide, infecção e rigidez $\operatorname{articular}^{(1,2,10)}$. O método visa à remoção da calcificação associada à bursectomia, com resultados promissores.

O objetivo deste trabalho é avaliar os resultados obtidos com o tratamento artroscópico de pacientes com tendinite calcária refratária do ombro e correlacionálos com a acromioplastia associada.

\section{MÉTODOS}

Trata-se de um estudo transversal de setembro de 2001 a junho de 2006, em que 55 pacientes com tendinite calcária do ombro, resistentes ao tratamento conservador, foram submetidos à ressecção da calcificação por via artroscópica. Todos foram operados pelo mesmo cirurgião. O seguimento variou de 12 a 70 meses (média de 31 meses).

A média de idade foi de 42 anos, variando de 30 a 64 anos; 44 pacientes eram do sexo feminino (80\%), sendo 37 ombros direitos (67,27\%), com dominância em 63,63\% dos casos. Ver características clínico-demográficas na Tabela 1.

A dor foi o principal sintoma; o tempo decorrido entre o início dos sintomas e a artroscopia foi, em média, de 38 meses, variando de cinco a 120 meses. A "lesão em framboesa”, descrita por Snyder ${ }^{(4)}$, que corresponde a uma mancha vascular (hiperemiada) na face articular do tendão supraespinal, foi encontrada em 56 \% dos pacientes (Tabela 1). 
Tabela 1 - Características clínico-demográficas

\begin{tabular}{|c|c|c|c|c|c|c|c|c|c|c|c|c|c|}
\hline Caso & Sexo & Dom & Lado & $\begin{array}{l}\text { Idade } \\
\text { (anos) }\end{array}$ & LF & Acrom & $\mathrm{Te}$ & Bosw & Gart & $\begin{array}{c}\text { Sutura } \\
\text { M R }\end{array}$ & $\begin{array}{c}\text { Seg } \\
\text { meses }\end{array}$ & UCLA & Compl \\
\hline 1 & $\mathrm{M}$ & & $E$ & 50 & & & SE & média & 1 & & 70 & 26 & Dor residual \\
\hline 2 & $\mathrm{~F}$ & + & $\mathrm{D}$ & 48 & & + & $\mathrm{IE}$ & pequena & II & & 69 & 31 & \\
\hline 3 & $\mathrm{~F}$ & + & $\mathrm{D}$ & 47 & + & & SE & média & 1 & & 67 & 30 & \\
\hline 4 & $\mathrm{~F}$ & + & $D$ & 39 & & & $\mathrm{IE}$ & pequena & 1 & & 66 & 35 & \\
\hline 5 & $\mathrm{M}$ & + & $\mathrm{D}$ & 49 & + & & SE & grande & 1 & & 61 & 35 & \\
\hline 6 & $\mathrm{~F}$ & + & $\mathrm{D}$ & 37 & & + & SE & pequena & 1 & & 52 & 31 & \\
\hline 7 & $\mathrm{M}$ & + & $\mathrm{D}$ & 45 & + & + & SE & média & III & & 50 & 35 & \\
\hline 8 & $\mathrm{M}$ & + & $\mathrm{D}$ & 40 & + & + & SE & grande & 1 & + & 49 & 34 & \\
\hline 9 & $\mathrm{M}$ & + & $\mathrm{D}$ & 30 & & + & SE & pequena & II & & 47 & 35 & \\
\hline 10 & $\mathrm{M}$ & & $E$ & 49 & + & + & SE & média & 1 & & 45 & 35 & \\
\hline 11 & $\mathrm{~F}$ & + & $\mathrm{D}$ & 38 & + & & SE & média & 1 & & 43 & 35 & \\
\hline 12 & $\mathrm{~F}$ & & $\mathrm{E}$ & 51 & + & + & SE & média & 1 & & 42 & 35 & \\
\hline 13 & $\mathrm{M}$ & & $\mathrm{E}$ & 64 & + & & SE & grande & II & & 41 & 35 & \\
\hline 14 & $M$ & + & $\mathrm{D}$ & 35 & + & & SE & média & 1 & & 41 & 35 & \\
\hline 15 & $\mathrm{~F}$ & + & $\mathrm{D}$ & 42 & + & + & $\mathrm{IE}$ & média & 1 & & 40 & 31 & Capsulite \\
\hline 16 & $\mathrm{~F}$ & + & $\mathrm{D}$ & 40 & & & SE & média & 1 & & 40 & 35 & \\
\hline 17 & $\mathrm{~F}$ & + & $\mathrm{D}$ & 38 & + & + & SE & média & $\mathrm{I}$ & & 38 & 35 & \\
\hline 18 & $\mathrm{~F}$ & + & $\mathrm{D}$ & 37 & + & & SE & média & 1 & & 38 & 35 & \\
\hline 19 & $\mathrm{~F}$ & & $E$ & 30 & + & & $\mathrm{IE}+\mathrm{SE}$ & média & 1 & & 36 & 35 & \\
\hline 20 & $\mathrm{~F}$ & + & $\mathrm{D}$ & 32 & & & SE & grande & 1 & & 35 & 35 & \\
\hline 21 & $\mathrm{M}$ & + & D & 35 & + & & SE & média & 1 & & 33 & 35 & \\
\hline 22 & $\mathrm{~F}$ & + & $\mathrm{D}$ & 50 & + & & SE & média & 1 & & 33 & 35 & \\
\hline 23 & $\mathrm{~F}$ & + & $\mathrm{D}$ & 50 & + & & SE & grande & II & & 33 & 31 & Capsulite \\
\hline 24 & $\mathrm{~F}$ & & $\mathrm{E}$ & 58 & + & & SE & pequena & II & & 32 & 35 & \\
\hline 25 & $\mathrm{M}$ & & $\mathrm{E}$ & 42 & + & & SE & pequena & 1 & & 31 & 35 & Capsulite \\
\hline 26 & $M$ & + & $\mathrm{D}$ & 40 & + & & SE & grande & 1 & & 30 & 35 & \\
\hline 27 & $\mathrm{~F}$ & + & $\mathrm{D}$ & 38 & + & & $\mathrm{IE}$ & grande & 1 & & 30 & 35 & Capsulite \\
\hline 28 & $\mathrm{~F}$ & + & $\mathrm{D}$ & 48 & & & $\mathrm{IE}$ & pequena & 1 & & 30 & 35 & \\
\hline 29 & $\mathrm{~F}$ & & $\mathrm{E}$ & 30 & + & & SE & média & II & & 29 & 35 & \\
\hline 30 & $\mathrm{~F}$ & + & $\mathrm{D}$ & 32 & + & & SE & média & 1 & & 28 & 35 & \\
\hline 31 & $\mathrm{~F}$ & & $\mathrm{E}$ & 33 & + & & SE & média & III & & 27 & 35 & \\
\hline 32 & $\mathrm{~F}$ & + & $D$ & 32 & & & SE & pequena & II & & 26 & 35 & \\
\hline 33 & $\mathrm{~F}$ & & $E$ & 55 & & & SE & média & 1 & & 25 & 35 & Capsulite \\
\hline 34 & $\mathrm{~F}$ & + & $\mathrm{D}$ & 37 & & & $\mathrm{IE}$ & média & 1 & & 25 & 35 & \\
\hline 35 & $\mathrm{~F}$ & & $\mathrm{E}$ & 52 & + & & SE & média & 1 & & 25 & 35 & \\
\hline 36 & $\mathrm{~F}$ & + & $\mathrm{D}$ & 45 & + & & SE & média & 1 & & 24 & 35 & \\
\hline 37 & $\mathrm{~F}$ & & $E$ & 60 & & & SE & média & II & & 24 & 35 & \\
\hline 38 & $\mathrm{~F}$ & + & $\mathrm{D}$ & 32 & & + & $\mathrm{IE}$ & pequena & II & & 23 & 35 & \\
\hline 39 & $\mathrm{~F}$ & + & $\mathrm{D}$ & 58 & & + & $\mathrm{IE}$ & pequena & II & & 22 & 35 & \\
\hline 40 & $\mathrm{~F}$ & & $E$ & 58 & & + & SE & pequena & II & & 22 & 32 & Capsulite \\
\hline 41 & $\mathrm{~F}$ & + & $\mathrm{D}$ & 40 & + & & SE & grande & 1 & & 20 & 35 & \\
\hline 42 & $\mathrm{~F}$ & & $E$ & 57 & & & SE & pequena & III & & 20 & 35 & \\
\hline 43 & $\mathrm{~F}$ & + & $\mathrm{D}$ & 45 & + & & SE & pequena & 1 & & 20 & 35 & \\
\hline 44 & $\mathrm{~F}$ & + & $D$ & 47 & & & $\mathrm{IE}$ & média & 1 & & 20 & 35 & \\
\hline 45 & $\mathrm{~F}$ & + & $\mathrm{D}$ & 50 & + & & SE & média & 1 & & 18 & 35 & \\
\hline 46 & $\mathrm{~F}$ & + & $\mathrm{D}$ & 45 & & & SE & média & II & & 15 & 34 & \\
\hline 47 & $\mathrm{~F}$ & & $E$ & 34 & & & SE & média & 1 & & 13 & 35 & \\
\hline 48 & $\mathrm{~F}$ & + & $\mathrm{D}$ & 50 & + & & SE & média & 1 & & 12 & 35 & \\
\hline 49 & $\mathrm{~F}$ & + & $D$ & 50 & & & $\mathrm{IE}$ & pequena & 1 & & 12 & 26 & Dor Residual \\
\hline 50 & $\mathrm{~F}$ & + & $\mathrm{D}$ & 40 & + & & SE & pequena & 1 & & 12 & 35 & \\
\hline 51 & $\mathrm{~F}$ & + & $\mathrm{D}$ & 46 & + & & $\mathrm{IE}+\mathrm{SE}$ & média & 1 & & 12 & 25 & Dor Residual \\
\hline 52 & $\mathrm{~F}$ & & $E$ & 50 & & & SE & grande & 1 & & 12 & 35 & \\
\hline 53 & $\mathrm{~F}$ & + & $\mathrm{D}$ & 42 & & & SE & grande & II & & 12 & 35 & \\
\hline 54 & $\mathrm{~F}$ & & $E$ & 37 & & & $\mathrm{IE}$ & média & 1 & & 12 & 35 & \\
\hline 55 & $\mathrm{~F}$ & & $\mathrm{E}$ & 38 & + & & SE & média & I & & 12 & 35 & \\
\hline
\end{tabular}

Fonte: Arquivos médicos do HOG

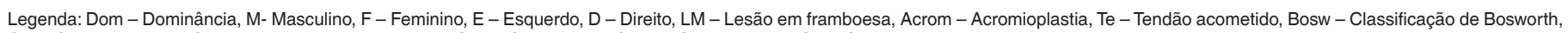
Gart- Classificação de Gartner, MR - Manguito rotador, Seg - Seguimento, Compl- Complicações, SE - Supraespinal, IE - Infraespinal 
Quanto aos tendões acometidos, o supraespinal em 42 casos, o infraespinal em 11 e a associação entre eles em dois casos (Figura 3). A acromioplastia foi realizada pela presença de atrito subacromial, observado pela fibrilação na superfície anteroinferior do acrômio ou quando não encontramos a calcificação no intraoperatório (Figura 4), enquanto a bursectomia subacromial foi realizada em todos os casos.

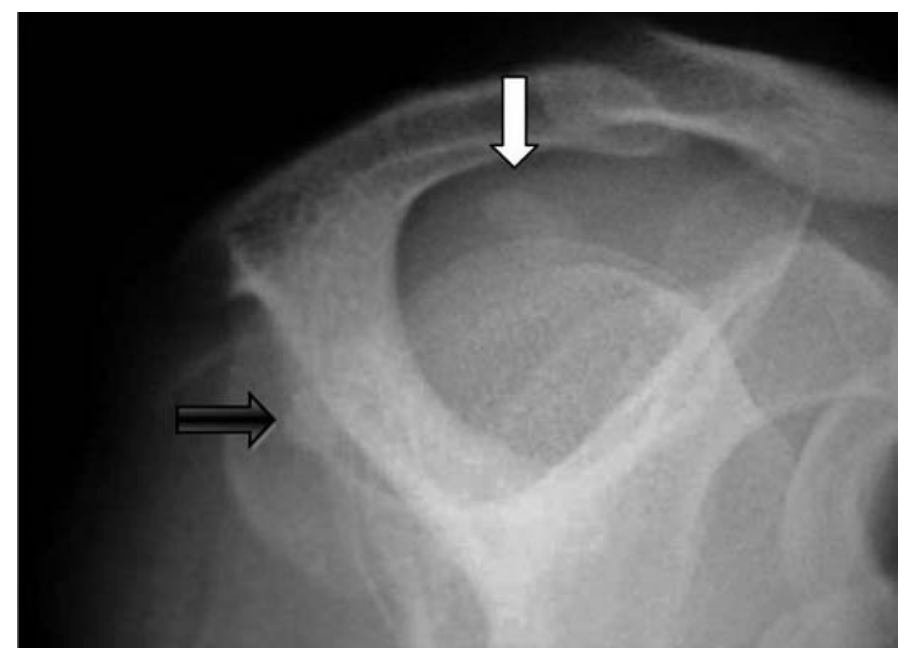

Figura 3 - Perfil escapular evidenciando calcificações no supraespinal (seta branca) e infraespinal (seta preta)

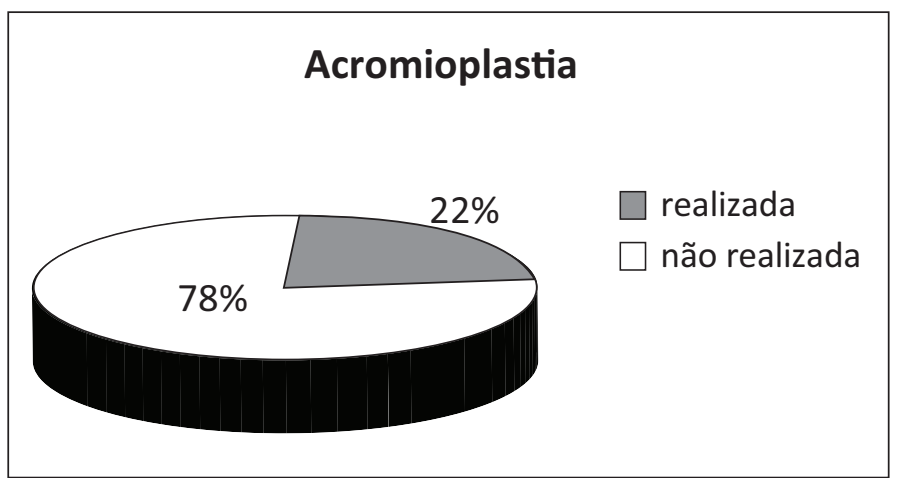

Figura 4 - Acromioplastia

A avaliação radiográfica foi realizada nas posições anteroposterior em rotação interna e externa, perfil escapular com $10^{\circ}$ de inclinação caudal e perfil axilar ${ }^{(1)}$. Os índices quanto à classificação de Bosworth ${ }^{(5)}$, que se baseia no tamanho da calcificação, e de Gartner ${ }^{(16)}$, relacionada com a densidade da mesma, estão expressos nas Figuras 5 e 6 . Entre os operados, apenas um paciente $(1,81 \%)$ teve o defeito tendíneo suturado, após a ressecção da calcificação (amostra insuficiente para análise estatística). Na série estudada não foram encontradas lesão $\mathrm{SLAP}^{(4)}$ nem rotura do manguito rotador associada.

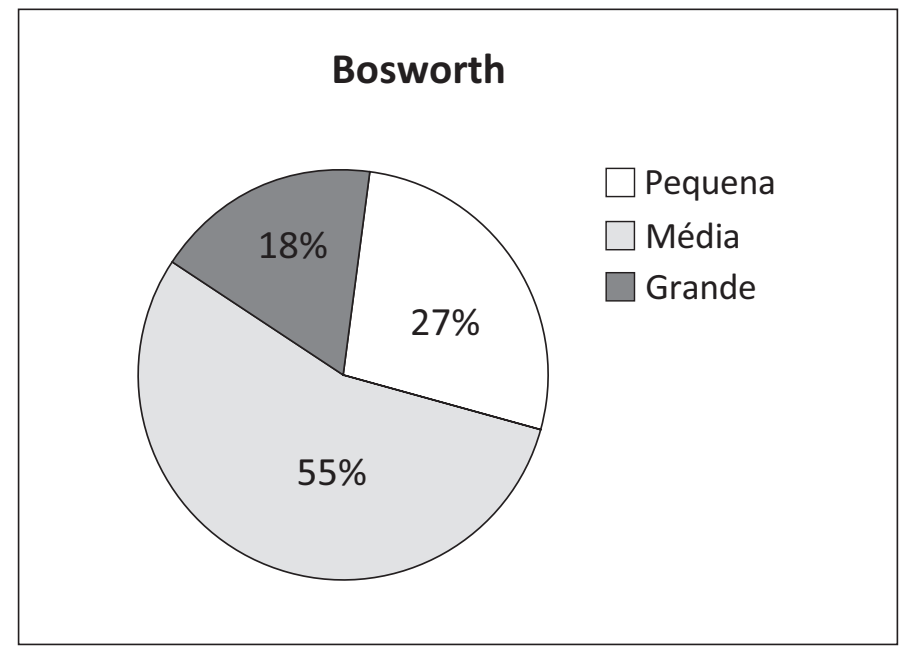

Figura 5 - Índices segundo a classificação de Bosworth

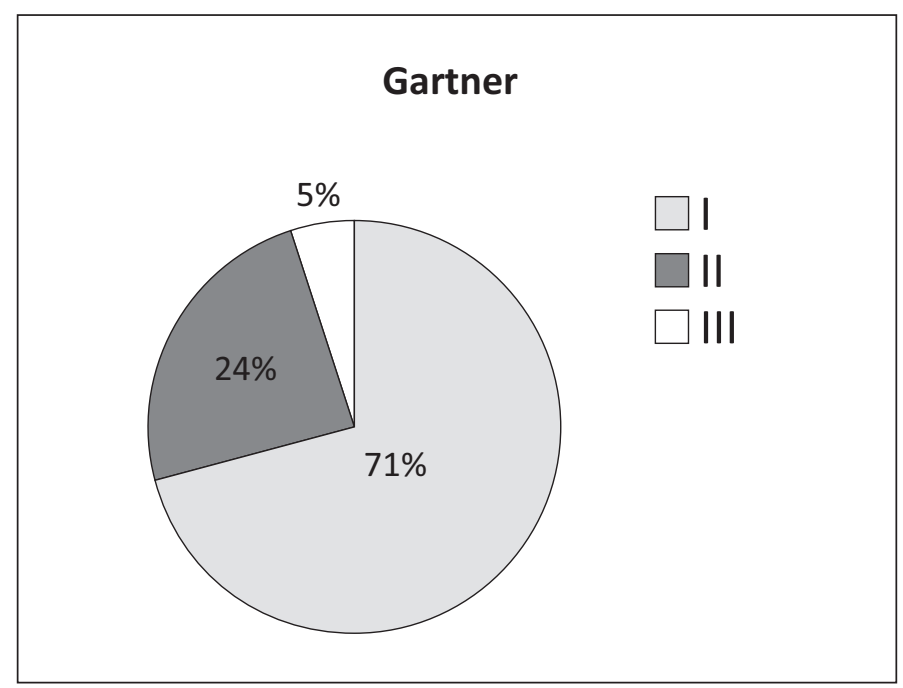

Figura 6 - Índices segundo a classificação de Gartner

A cirurgia artroscópica foi realizada em decúbito lateral, com os portais posterior, lateral e anterior. Nas últimas 35 cirurgias, não foi utilizado o portal anterior (Figura 7), onde a irrigação passou a ser realizada pelo portal lateral, assim como a instrumentação. Importante demarcar com um fio de sutura monofilamentar a face articular do tendão supraespinal na zona de hiperemia (Figura 8), quando existente, que pode corresponder à área da calcificação na face bursal ${ }^{(4)}$. É fundamental a remoção completa da calcificação sem causar dano iatrogênico no tendão acometido (Figura 9), assim como irrigação exaustiva do espaço subacromial, pois resíduos de cálcio (Figura 10) podem causar dor no pós-operatório ${ }^{(2)}$. Não foi utilizado o intensificador de imagem durante o ato cirúrgico. Os pacientes foram avaliados pelo sistema proposto pela University of $\mathrm{Ca}$ lifornia at Los Angeles-UCLA ${ }^{(17)}$. 


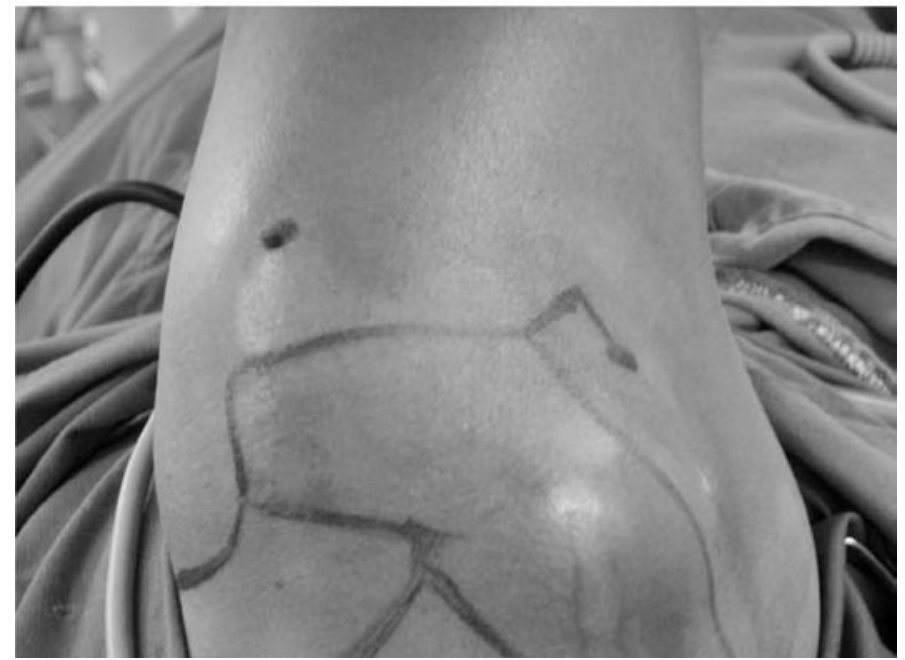

Figura 7 - Portais artroscópicos posterior e lateral em decúbito lateral do ombro direito

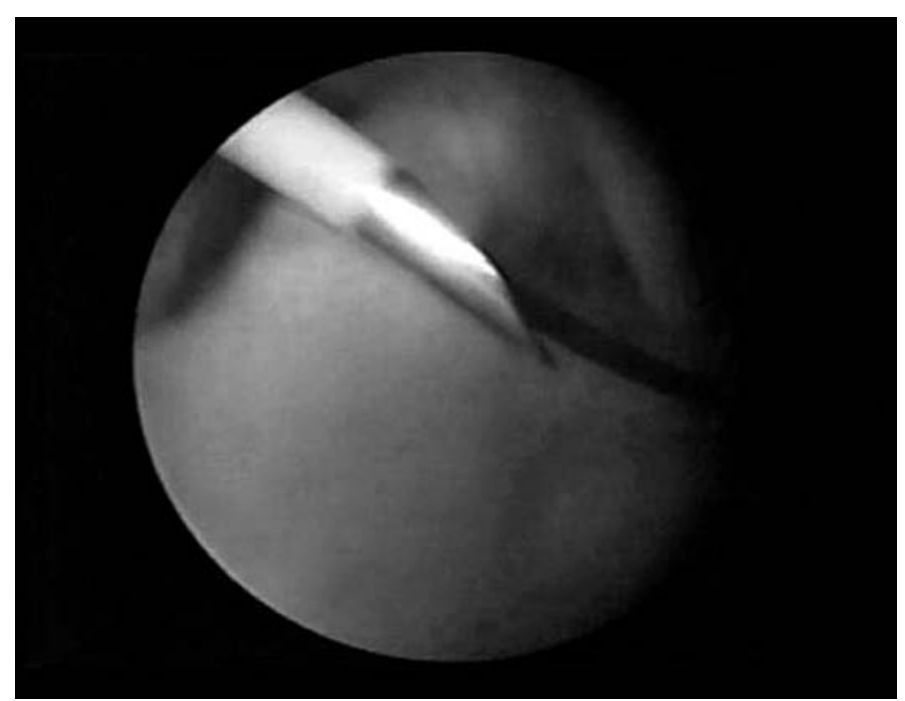

Figura 8 - Fio-guia na face articular do tendão supraespinal de ombro direito, vista pelo portal posterior

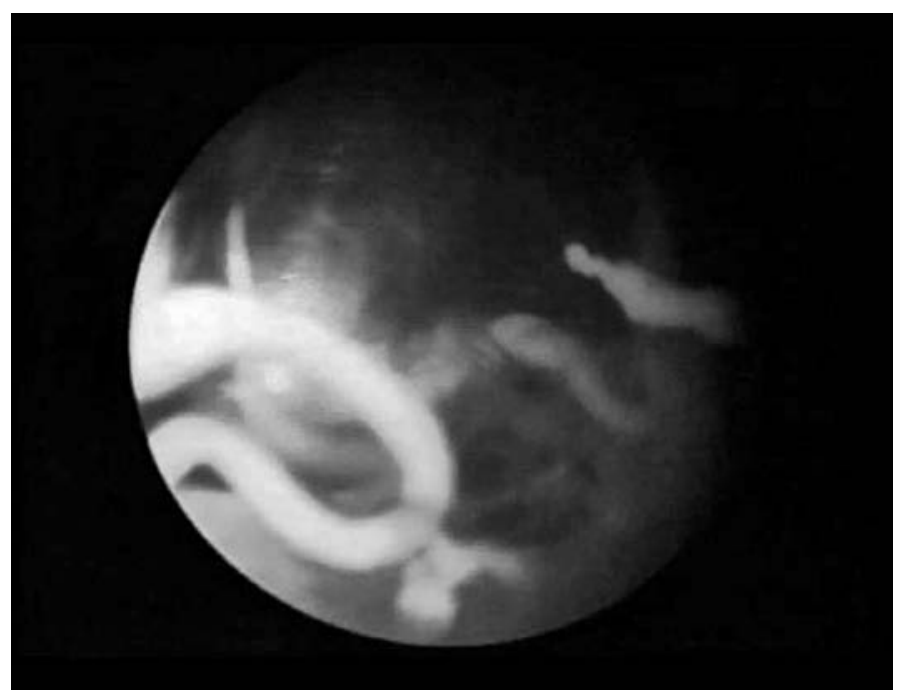

Figura 9 - Remoção da calcificação vista pelo portal posterior

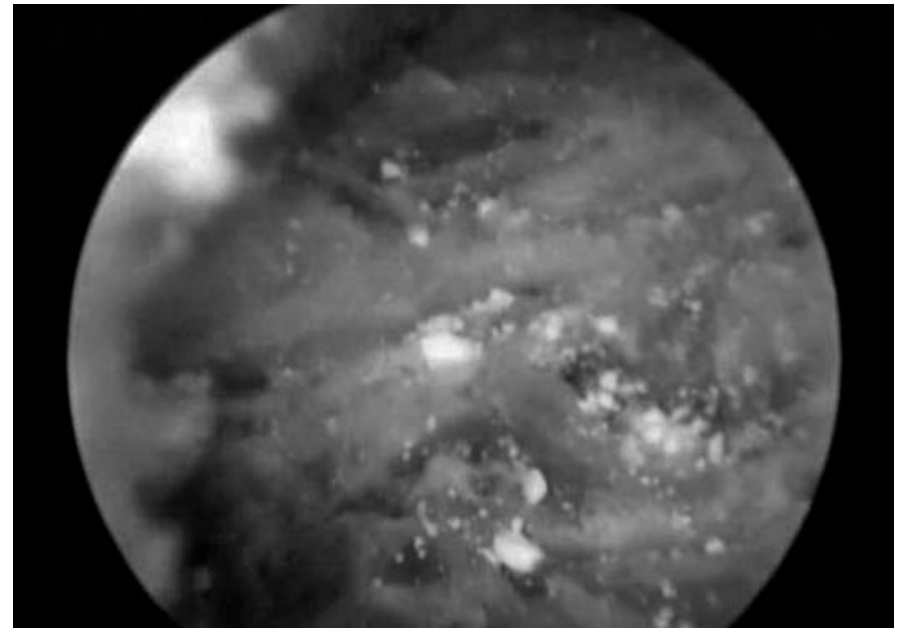

Figura 10 - Presença dos grânulos calcários no espaço bursal, vistos pelo portal posterior

Os dados foram analisados através do software SSPS 11.5 (Statistical Package for the Social Sciences). Foi realizado o teste da análise de variância para verificar se existe diferença entre a acromioplastia e as médias da variável contínua idade; teste de Fisher quando se propôs a comparação das variáveis discretas 2x2 e o teste do qui-quadrado quando se verificaram diferenças ou não entre as variáveis discretas com mais de dois grupos. Utilizou-se o nível de confiança de 95\%, ou seja, significativo se $\mathrm{p}<0,05$.

\section{RESULTADOS}

Segundo os critérios da UCLA, foram encontrados 46 resultados excelentes e seis bons, totalizando 52 resultados satisfatórios (94,54\%). Os três casos insatisfatórios (regulares) ocorreram pela dor residual pós-operatória, mesmo após a reabilitação, porém sem limitação articular. Seis pacientes evoluíram com capsulite adesiva no pós-operatório precoce, todos solucionados com bloqueios seriados do nervo supraescapular.

Em quatro pacientes não foi encontrada a calcificação; em três desses foi realizada a acromioplastia e em um, não.

Nenhum caso de infecção, lesão nervosa ou dor acromioclavicular foi encontrado.

Não existe diferença significativa entre os pacientes que realizaram ou não a acromioplastia, em relação à idade $(\mathrm{p}=0,851)$ (Tabela 2$)$; em relação ao sexo ( $\mathrm{p}=0,133)$; presença ou não da "lesão em framboesa" $(p=0,209)$; dominância $(p=0,231)$ e lado $(0,231)$. Não houve relação também entre o tamanho e o tipo da calcificação com a variável discreta acromioplastia ( $p=0,219$ e $p=0,193$, respectivamente). 
Tabela 2 - Correlação das variáveis acromioplastia $\times$ idade

\begin{tabular}{c|c|c|c}
\hline \multirow{2}{*}{ Variável } & \multicolumn{2}{|c|}{ Acromioplastia } & \multirow{2}{*}{} \\
\cline { 2 - 3 } & Sim & Não & \multirow{2}{*}{$\mathbf{p}$} \\
\cline { 2 - 3 } & Média \pm DP & Média \pm DP & \\
\hline Idade (anos) & $43,46 \pm 8,55$ & $44,00 \pm 9,18$ & 0,851 \\
\hline
\end{tabular}

Teste: Análise de variância

Pela Tabela 3 podemos afirmar que não houve diferença significativa entre os resultados satisfatórios encontrados pelo índice da UCLA e a realização ou não da acromioplastia $(\mathrm{p}=0,086)$. Em relação à classificação de Bosworth/Gartner e a avaliação da UCLA (Tabela 4).

Tabela 3 - Distribuição dos pacientes quanto à acromioplastia $\times$ UCLA

\begin{tabular}{c|c|c}
\hline \multirow{2}{*}{ UCLA } & \multicolumn{2}{|c}{ Acromioplastia } \\
\cline { 2 - 3 } & Não & Sim \\
\hline Excelente & 38 & 8 \\
\hline Bom & 3 & 3 \\
\hline Regular & 2 & 1 \\
\hline Total & 43 & 12 \\
\hline
\end{tabular}

$P=0,086 /$ Teste de Fisher

Tabela 4 - UCLA $\times$ Bosworth / UCLA $\times$ Gartner

\begin{tabular}{c|c|c|c|c|c|c}
\hline \multirow{2}{*}{ Classificação } & \multicolumn{6}{c}{ UCLA } \\
\cline { 2 - 7 } & \multicolumn{2}{|c|}{ Bom } & \multicolumn{2}{c}{ Excelente } & \multicolumn{2}{c}{ Regular } \\
\cline { 2 - 7 } & n. & $\%$ & n. & $\%$ & n. & $\%$ \\
\hline Bosworth & & & & & & \\
Pequena & 2 & 33,3 & 11 & 23,9 & 2 & 66,7 \\
Média & 3 & 50,0 & 26 & 56,5 & 1 & 33,3 \\
Grande & 1 & 16,7 & 9 & 19,6 & - & 0,0 \\
\hline Gartner & & & & & & \\
I & 2 & 33,3 & 33 & 71,7 & 2 & 66,7 \\
II & 4 & 66,7 & 10 & 21,7 & 1 & 33,3 \\
III & - & 0,0 & 3 & 6,5 & - & 0,0 \\
\hline
\end{tabular}

\section{DISCUSSÃO}

A tendinite calcária é uma doença autolimitada ${ }^{(1,3,5)}$. É importante entendermos que a reabsorção da calcificação ocorrerá, mas quando? Talvez essa seja a verdadeira variável em todo o processo. Recomenda-se sempre, inicialmente, o tratamento conservador, antes de se pensar em tratamento cirúrgico. Na nossa casuística, o tempo decorrido entre o início dos sintomas e a artroscopia foi, em média, de 38 meses, com mínimo de cinco meses. $\mathrm{O}$ grande cuidado que se deve ter é o alto índice de reabsorção espontânea que essa patologia apresenta.
Muito se tem publicado sobre a utilização da artroscopia no tratamento das lesões do ombro. Essa técnica já conquistou seu espaço, tendo se mostrado eficiente no tratamento da tendinite calcária do ombro, refratária à conduta conservadora, apresentando menor morbidade e possibilitando ao paciente retorno mais precoce às suas atividades diárias ${ }^{(2,4,10-15)}$. Por sua vez, a cirurgia aberta apresenta problemas com a inserção do músculo deltoide, infecção, maior rigidez articular e dificuldade de visualização $\operatorname{articular}^{(3,8)}$.

Em nosso trabalho, os resultados satisfatórios somaram 94,54\%, coincidindo com os da literatura. Godinho et al tiveram 94\% de excelentes e bons resultados ${ }^{(2)}$, Bassini et $a l^{(18)} 88,9 \%$, Ark et al ${ }^{(12)} 91 \%$ e Molé et $a l^{(11)} 89 \%$.

Em concordância com as publicações ortopédicas, observamos em nossos resultados maior incidência da tendinite calcária em pacientes do sexo feminino, com a maioria na faixa etária entre 30 e 50 anos e com predomínio do lado dominante ${ }^{(2,3,10,12)}$. A avaliação radiográfica é importante para a classificação e localização da calcificação, sendo necessário um estudo radiográfico no pré-operatório imediato, para que não ocorra a reabsorção da calcificação entre a indicação e a cirurgia ${ }^{(1,2)}$.

Segundo Godinho et $a l^{(2)}$, Ark et $a l^{(12)}$ e Rupp et $a l^{(19)}$, a ressecção parcial ou total da calcificação não influencia os resultados satisfatórios do tratamento artroscópico. Pensamos sempre que a ressecção deva ser a mais completa possível, o que concorda com Checchia et $a l^{(10)}$ e Jerosch et $a l^{(15)}$, apesar de nem sempre ser fácil essa avaliação no intraoperatório ${ }^{(18)}$. Porcellini et $a^{(20)}$ observaram que a presença de resíduos calcários no pós-operatório diminuiu significativamente o escore “dor” do índice de Constant-Murley, em relação àqueles com remoção completa. No presente estudo, não se utilizou o intensificador de imagem durante o ato cirúrgico, ao contrário de Checchia et al, que certificaram se a ressecção calcária tinha sido completa ${ }^{(10)}$. Adicionalmente, se houver dúvidas sobre a remoção da calcificação, pode-se realizar uma radiografia na mesa cirúrgica ${ }^{(4)}$.

Há autores que defendem a não sutura da abertura do tendão, após a ressecção da calcificação ${ }^{(8,20)}$. Procura-se, sempre que possível, não incisar o tendão, utilizandose de uma agulha percutânea lateral ao acrômio para as perfurações do mesmo, auxiliado por uma cureta artroscópica. Não se deve passar a lâmina de shaver 
sobre o tendão para se evitar um dano iatrogênico e sim utilizá-la como uma sucção a vácuo da calcificação, de preferência, lâminas synovial resector de pequeno diâmetro $^{(4)}$.

Na nossa série, foi realizada apenas uma sutura do manguito (1,81\%), pois não tivemos pacientes que, após a ressecção, ficaram com lesões parciais grandes ou completas e dos 54 ombros que não tiveram os defeitos suturados, 94\% alcançaram resultados satisfatórios. A amostra de um paciente com sutura foi insuficiente para uma análise estatística. Porcellini et $a^{(20)}$ referem que não houve sutura do tendão em lesões longitudinais parciais menores que $1 \mathrm{~cm}$, o que correspondeu a $38,1 \%$ e no seu seguimento não houve rotura do manguito rotador, tanto nos pacientes que fizeram sutura como naqueles que não fizeram. Afirmam ainda que não suturam, quando a remoção é incompleta. Na serie de 71 ombros de Checchia et al ${ }^{(10)}, 17$ (23,9\%) tiveram os defeitos tendíneos suturados, sendo que dos 54 que não receberam sutura, 51 obtiveram resultados satisfatórios, correspondendo a $94,44 \%$. Pode-se sugerir que tal procedimento somente se faz necessário em defeitos grandes. Sempre que precisar incisar o tendão, sempre fazer no sentido longitudinal de sua fibras e não transversal, para se evitar tensão na sutura, o que comprometeria a reabilitação.

A acromioplastia se faz necessária? $\mathrm{Neer}^{(21)}$, em 1990, afirmou que não, pois a tendinite calcária não está associada a impacto subacromial. Ellman et al ${ }^{(17)}$ recomendam realizá-la somente na evidência de sinais de atrito. Em um estudo multicêntrico francês, 112 pacientes com tendinite calcária foram operados artroscopicamente com e sem descompressão subacromial e concluiu-se não haver diferenças estatísticas significativas entre os dois grupos ${ }^{(11)}$. Mold et $a l^{(22)}$, também em estudo prospectivo randomizado de 74 pacientes com e sem acromioplastia, afirmaram que essa variável não melhora o resultado final. Jerosch et $a^{(15)}$ também não verificaram nenhum benefício da acromioplastia e Snyder somente realiza a descompressão concomitante em $20 \%$ dos seus pacientes aproximadamente ${ }^{(4)}$.

Checchia et $a^{(10)}$ realizaram acromioplastia em 90,2\% dos casos com acrômio tipo II e III ${ }^{(21)}$ e na presença radiográfica ou intraoperatória de impacto, por acreditar que diminua a dor no pós-operatório. Godinho et $a^{(2)}$ efetuaram em $89,1 \%$ dos pacientes com pinçamento subacromial, evolução longa, acrômio III e ressecção parcial e concluíram que a acromioplastia é desnecessária. Na nossa série, não foi realizada descompressão subacromial em 43 pacientes, pois o acrômio se mostrou liso e brilhante no intraoperatório, na grande maioria dos casos, demonstrando não haver qualquer tipo de atrito subacromial. A acromioplastia se faz necessária quando há impacto ou quando não se encontra a calcificação. Em 43 casos sem acromioplastia, 42 se mostraram satisfatórios, o que pode sugerir que a acromioplastia não participa rotineiramente da técnica cirúrgica $(p=0,086)$. Não há relação entre essa variável e o tamanho e tipo da calcificação ( $\mathrm{p}=0,219$ e $\mathrm{p}=0$,193). Sistematicamente, não liberamos o ligamento coracoacromial por ser um importante contensor da cabeça umeral e não um fator prognóstico do sucesso da técnica.

A dor residual esteve presente em três pacientes no pós-operatório, os quais foram tratados com reabilitação e todos evoluíram para resultados regulares. A complicação mais comum foi a capsulite adesiva, também presente em estudos de Godinho et $a l^{(2)}$ e Checchia et $a l^{(10)}$. Foram identificados seis casos, tratados com bloqueios do nervo supraescapular e todos evoluíram com resultados satisfatórios, segundo o índice da UCLA. Nenhum paciente apresentou migração do depósito calcário para localização intraóssea ${ }^{(23)}$, como também não houve casos de dor na articulação acromioclavicular ${ }^{(10)}$.

Nas últimas 35 artroscopias da série, utilizamos apenas dois portais: o posterior e o lateral. Por ser um procedimento rápido na sua execução, deixamos de realizar o portal anterior para irrigação, já que melhora a cosmese pós-operatória e não dificulta em nada o ato cirúrgico. A irrigação, então, passa a ser feita pelo portal lateral, assim como a instrumentação.

Remoção parcial ou completa da calcificação? Realização ou não da acromioplastia? O defeito tendíneo do manguito rotador deve ou não ser suturado? Essas continuam sendo as variáveis mais discutidas na literatura, tanto em estudos descritivos quanto em ensaios clínicos randomizados do assunto: tratamento cirúrgico da tendinite calcária do ombro.

\section{CONCLUSÃO}

O tratamento artroscópico da tendinite calcária do ombro sugere ter altos índices de resultados satisfatórios, sendo, portanto, um método eficaz. A acromioplastia não se faz necessária e não melhora os resultados. 


\section{REFERÊNCIAS}

1. Zoppi AF. Tendinite calcária. In: Godinho GG. Clínica ortopédica - Atualização em cirurgia do ombro. Rio de Janeiro: Medsi; 2000. p. 111-6.

2. Godinho GG, Freitas JMA, Vieira AW, Antunes LC, Castanheira EW. Tratamento artroscópico da tendinite calcária do ombro. Rev Bras Ortop. 1997;32(9):669-74.

3. De Palma AF. Calcareous tendinitis. In: Surgery of the shoulder. 3rd ed. Philadelphia: Lippincott; 1983. p.277-85.

4. Snyder SJ. Shoulder arthroscopy. New York: McGraw Hill; 1993.

5. Bosworth BM. Examination of the shoulder for calcium deposits. Technique of fluoroscopy and spot film roentgenography. J Bone Joint Surg. 1941;23:567-77.

6. Welfling J, Kahn MF, Desroy M, Paolaggi JB, de Sèze S. Les calcifications de l'epaule II. La maladie des calcifications tendineuses multiples. Rev Rhum Mal Osteoartic. 1965;32(6):325-34.

7. Sandstrom C, Wahlgren F. Peritendinitis calcarea: common disease of middle life: its diagnosis, pathology and treatment. AJR 1938;40:1-21

8. Uhthoff HK, Sarkar K. Calcifying tendonitis. In: Rockwood CA Jr, Matsen FA 3rd. The shoulder. Philadelphia: Saunders; 1990. p.147-9.

9. Rompe JD, Zoellner J, Nafe B. Shock wave therapy versus conventional surgery in the treatment of calcifying tendinitis of the shoulder. Clin Orthop Relat Res. 2001;(387):72-82.

10. Checchia SL, Miyazaki AN, Fregoneze M, Santos PD, Silva LA, Mazza LFF, et al. Tratamento artroscópico da tendinite calcária do ombro. Rev Bras Ortop. 2007;42(6):161-8.

11. Molé D, Kempf JF, Gleyze P, Rio B, Bonnomet F, Walch G. Results of endoscopic treatment of non-broken tendinopathies of the rotator cuff. Rev Chir Orthop Reparatrice Appar Mot. 1993;79(7):532-41.

12. Ark JW, Flock TJ, Flatow EL, Bigliani LU. Arthroscopic treatment of calcific tendinitis of the shoulder. Arthroscopy. 1992,8(2):183-8.

13. Tilander BM, Norlin RO. Change of calcifications after arthroscopic subacromial decompression. J Shoulder Elbow Surg. 1998;7(3):213-7.

14. Ozkoc G, Akpinar S, Hersekli MA, Ozalay M, Tandogan RN. Arthroscopic treatment of rotator cuff calcifying tendinitis. Acta Orthop Traumatol Turc. 2002;36(5): 13-6.

15. Jerosch J, Strauss JM, Schmiel S. Arthroscopic treatment of calcific tendinitis of the shoulder. J Shoulder Elbow Surg. 1998;7(1):30-7.

16. Gartner J. Tendinosis calcarea: results of treatment with needling. Z Orthop Ihre Grenzgeb. 1993;131(5):461-9.

17. Ellman H, Hanker G, Bayer M. Repair of the rotator cuff. End result study of factors influencing reconstruction. J Bone Joint Surg Am. 1986;68(8): 1136-44.

18. Bassini F, Rescazzi R, Murena PF. Arthroscopic treatment of the calcifying tendinitis of the rotator cuff. J Shoulder Elbow Surg. 1995;4(1):526.

19. Rupp S, Khon D, Litzenburger H, Seil R. Arthroscopic treatment of chronically painful calcifying tendinitis of the supraespinatus tendon. Arthroscopy. 2006;22(5):521-7.

20. Porcellini G, Paladini P, Campi F, Paganelli M. Artroscopic treatment of calcifying tendinitis of the shoulder. Clinical and ultrasonografic follow-up findings at two to five years. J Shoulder Elbow Surg. 2004;13(5):503-8.

21. Neer CS. Calcium deposit. In: Shoulder reconstruction. Philadelphia: WB Saunders; 1990. p.427-3.

22. Mold D, Gonzalvez M, Rio B. Arthroscopic treatment of calcifying tendinitis: is acromioplasty useful? J Shoulder Elbow Surg. 1995;4(1):519.

23. Seyahi A, Demirhan M. Arthroscopic removal of intraosseous and intratendinous deposits in calcifying tendinitis of the rotator cuff. Arthroscopy. 2009;25(6):590-6. 